\title{
Optimization of in-situ Nanoparticulate Gel of Ofloxacin using Factorial Design to Improve Treatment Strategy for Conjunctivitis and Corneal Ulcers
}

\author{
Akhil Sharma ${ }^{1, *}$, Shaweta Sharma ${ }^{2}$, Dimple Singh Tomar ${ }^{3}$ \\ ${ }^{1}$ R.J College of Pharmacy, Raipur, Aligarh, Uttar Pradesh, INDIA. \\ ${ }^{2}$ Galgotias University, Gautam Buddh Nagar, Uttar Pradesh, INDIA. \\ 3IIMT College of Pharmacy, Greater Noida, Uttar Pradesh, INDIA.
}

\begin{abstract}
Background: One of the main problems in ophthalmic drug delivery is the rapid elimination of conventional liquid eye drops from the eye due to rapid tear turnover resulting precorneal loss because of irritation caused by the drug preparation from large volume of the administered eye drop $(\sim 50 \mu \mathrm{l})$, a very small amount i.e. $<5 \%$ of the dosage actually penetrates and is able to reach intraocular tissues. Ofloxacin (OFL), a fluoroquinolone drug is used for the treatment of conjunctivitis and corneal ulcers and it is given quarterly in a day because its bioavailability is very low. The present study was designed to increase the ocular residence time of drug by formulating nanoparticulate in-situ gel for ensuring low irritation, better release and compatibility with ocular tissue. Materials and Methods: Initially, OFL nanoparticles were prepared using single emulsion solvent evaporation method and evaluated for various evaluation parameters. Based on the results, an optimized batch (B3) was selected by applying factorial design; which was further converted into in-situ gel. Results: Results of evaluation parameters revealed that optimized batch (B3) showed significant $(p=0.0001$ and $p=0.0090)$ increased in particle size and entrapment efficiency in comparison to rest batches. Moreover, nanoparticulate in-situ gel showed satisfactory results. Conclusion: Hence, Ofloxacin nanoparticulate in-situ gel thus formulated showed controlled drug release with lesser dosing frequency.
\end{abstract}

Key words: Ofloxacin, Nanoparticle(s), Nanoparticulate in-situ gel, Conjunctivitis, Factorial design.

\section{INTRODUCTION}

Controlled drug delivery provides the drug at a predetermined rate, locally or systemically for a specified period of time. ${ }^{1}$ Ocular drug delivery is one of the most appealing and arduous endeavours faced by the pharmaceutical scientists. Usually less than 5\% of the topically applied drug penetrates the cornea and attains the posterior segment of the eye. The bioavailability of an instilled conventional drug onto the ocular surface is usually low due to physiological mechanisms like tear drainage, protein binding, systemic absorption, enzymatic degradation and complex penetration barriers. Moreover, a bigger portion of it is absorbed systemically through the nasolacrimal duct which may give rise to systemic adverse effects. ${ }^{2}$ Nanotechnology deals with nanometer sized objects e.g. nanoparticles which are used for various reasons. ${ }^{3,4}$ As a drug delivery system, the in-situ gel has an advantage related to the gel or polymer network being formed (in-situ) providing sustained release of the drug. ${ }^{5}$ Ofloxacin (OFL) (( \pm )-9-fluro2, 3 dihydro-3-methyl-10 [4-methyl-1-piperazynyl-7-oxo-7H-pyrido [1, 2, 3-de]-1, 4-benzoxacine-6-carboxilic acid, stops or prevents ocular bacterial infections by either
Submission Date: 04-01-2019; Revision Date: 27-08-2019; Accepted Date: 06-11-2019

DOI: 10.5530/ijper.54.2.33 Correspondence: Dr. Akhil Sharma,

R.J College of Pharmacy, Raipur, Aligarh-202001, Uttar Pradesh, INDIA.

Phone: +919481687878

E-mail:xs2akhil@gmail.com

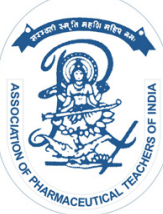

www.ijper.org 
killing or inhibiting their growth. ${ }^{6,7}$ It is reported that maximum serum concentrations after ten days of topical ophthalmic dosing were approximately 1000 times lower than standard oral doses of OFL. ${ }^{8}$ That's why, the present work aimed at formulation of an optimized Nano particulate in-situ ocular gel of Ofloxacin that would augment its ocular bioavailability and thus therapeutic adequacy which is vital for legitimate treatment of Conjunctivitis and Corneal Ulcers as well as such other ailments.

\section{MATERIALS AND METHODS Materials}

Various materials i.e. drug sample, additives, reagents etc. were obtained from different reputed companies. Ofloxacin-Vivan Life Sciences, Mumbai, Ethyl cellulose (EC)- Fine Chem Labs, Mumbai, DichloromethaneRankem, Ethanol- Jiangsu Huaxi International, Span -80- Rankem, Mannitol- Local source suppliers, Poly Vinyl Alcohol (PVA)- West coast laboratories, Mumbai, Gellan gum- Hi media GGM2388, Benzalkonium chloride, Sodium chloride, Sodium bicarbonate and Calcium chloride dihydrate- CDH Delhi, respectively.

\section{Methods}

\section{Estimation of $\lambda_{\max }$}

A sample $(5 \mu \mathrm{g} / \mathrm{ml})$ was scanned at the range of 200 $400 \mathrm{~nm}$ to access the $\lambda_{\max }$ value for OFL which was reported and confirmed by obtaining the overlain UV spectra of the drug using different concentrations (5-25 $\mu \mathrm{g} / \mathrm{ml})$.

\section{Drug-excipient compatibility study}

\section{Compatibility study using FTIR technique}

Drug-excipient compatibility study was carried out by FTIR (Shimadzu, Affinity-1) spectrophotometry. The mixture of drug and $\mathrm{KBr}$ (Potassium bromide) was ground into fine powder using mortar pestle and then compressed into discs in a hydraulic press at a pressure of $75 \mathrm{~kg} / \mathrm{cm}^{2}$. Each $\mathrm{KBr}$ disc was scanned 45 times at a resolution of $2 \mathrm{~cm}^{-1}$. The characteristic peaks were recorded and compared with that obtained with individual excipient used.

\section{Thin Layer Chromatographic (TLC) method}

The drug-excipient compatibility was also studied by densitometric TLC evaluation. The spots of drug and different excipients were obtained on pre-coated silica gel 60GF ${ }^{254}$ plates against n-butanol: ethanol: ammonia in the ratio of 5:5:4 $(\% \mathrm{v} / \mathrm{v})$ as mobile phase. The den- sitometric evaluation of separated spot was performed at $295 \mathrm{~nm}^{9}$

\section{Method of Preparation of Ofloxacin loaded nanoparticles}

Ofloxacin (OFL) loaded nanoparticles were prepared using single emulsion solvent evaporation method as given by Huyang Yan et al. with some modifications. Drug and polymer in different proportions were weighed and co-dissolved at room temperature into a mixture of ethanol and dichloromethane $(2: 1 \% \mathrm{v} / \mathrm{v})$ with magnetic stirring. This was slowly poured drop wise into the dispersion medium consisted of $20 \mathrm{ml}$ of $1 \%(\mathrm{w} / \mathrm{v})$ aqueous PVA and $1.5 \%(\mathrm{w} / \mathrm{v})$ span 80. Sonication was done by probe sonicator (PCI analytics, Mumbai (DP 120), MHA) for 2 min. on the ice bath. Later on, the system was put on magnetic stirrer (overnight) for complete evaporation of organic solvents. The prepared suspension was centrifuged (Remi, Mumbai, MHA) at $15000 \mathrm{rpm}$ for $1 \mathrm{hr}$. The supernatant was removed and the sediment was freeze dried in the presence of 5\% mannitol as cryoprotectant for $48 \mathrm{hrs}$ to stabilize the nanoparticles. The obtained particles were kept in dehydrated condition. ${ }^{10}$

\section{Evaluation of Nanoparticles}

The nanoparticles thus prepared were evaluated for various physicochemical parameters i.e. Percentage yield, Drug entrapment efficiency, Surface morphological study, Particle size and Zeta potential, in-vitro release and release kinetic studies.

\section{In-vitro release studies}

Drug-loaded nanoparticles were suspended in $\mathrm{pH} 7.4$ phosphate buffer in a glass vial which was placed in a mechanical shaking bath (100 cycles/min) at a temperature adjusted to $37^{\circ} \mathrm{C}$. At selected time intervals, samples were removed, replaced with fresh buffer medium and centrifuged at 15,000 rpm. The obtained supernatant was analyzed using U.V.-visible spectrophotometer at every transaction. ${ }^{10}$

\section{Factorial design}

Full factorial design was used in the present study. In this design 2 factors namely amount of EC (X1) and the polyvinyl alcohol (X2) were evaluated, at 3 levels $(-1,0,1)$ and experimental trials were performed at all 9 possible combinations. The amount of EC (X1) and PVA (X2) were chosen as independent while particle size and $\%$ entrapment, as dependent variables. The simplified models were then utilized to produce three dimensional response surfaces and contour plots to analyze the influence of independent variables. ${ }^{11}$ 


\section{Release kinetic}

The in-vitro dissolution data of Ofloxacin thus obtained was fitted into various pharmacokinetic models i.e. zero order, first order, higuchi and korsemeyer-peppas models to assess the release mechanism of drug.

\section{Preparation of Nano particulate in-situ gel}

Based on the results obtained from statistical studies, optimized batch was converted into gel with selected gelling agent.

\section{Determination of gelling capacity of selected gelling agents}

Aqueous solution of different concentration of polymer (s) i.e. Gallen gum, HPMC and Carbopol 940 with formulation codes F1, F2.........F6 were prepared and evaluated for their gelling capacity and viscosity in order to identify the compositions most suitable for use in in-situ gelling system. The gelling strength of prepared formulation (in-situ gel) was determined by placing 100 $\mathrm{ml}$ of the system in a vial containing $2 \mu \mathrm{l}$ of simulated tear fluid (STF) freshly prepared and equilibrated at $37^{\circ} \mathrm{C}$ and visually assessing the gel formation and noting the time for gelation including the time taken for the formed gel to dissolve (Table 1).

\section{Preparation of Nano particulate in-situ gel of Ofloxacin}

Nano particulate in-situ gel of Ofloxacin was prepared by using gellan gum because it showed highest viscosity that persists for longer duration of time and thus selected for further work. Gellan gum was dispersed in deionized water, heated to $90^{\circ} \mathrm{C}$ while stirring and then cooled to room temperature. Later on, drug loaded nanoparticles (equivalent to the prescribed dose of the drug) were dissolved in purified water and allowed with the addition of benzalkonium chloride. Then this solution was added to the above solution. The $\mathrm{pH}$ was adjusted in the range of 5.0-6.0 using $0.1 \mathrm{M} \mathrm{NaOH}$ Solution. The volume was made-up to $100 \mathrm{ml}$ with purified water. Prepared formulations were sterilized in an autoclave at $121^{\circ} \mathrm{C}$ and $15 \mathrm{psi}$ for $20 \mathrm{~min}$. (Table 2). ${ }^{12}$

\section{Evaluation of Nano particulate in-situ gel of Ofloxacin}

\section{Clarity}

The clarity before and after gelling was determined by visual examination of the formulations under light, alternatively against white and black backgrounds.

\section{pH}

Formulations were taken in a beaker and $\mathrm{pH}$ was checked using digital $\mathrm{pH}$ meter.

\section{Gelling strength}

The gelling strength of prepared formulation (in-situ gel) was determined by placing $100 \mathrm{ml}$ of the system in a vial containing $2 \mu \mathrm{l}$ of Simulated Tear Fluid (STF) freshly prepared and equilibrated at $37^{\circ} \mathrm{C}$ and visually assessing the gel formation and noting the time for gelation including the time taken for the formed gel to dissolve.

\section{Rheological evaluation}

Viscosity of formulation was determined after gelation using Brookfield viscometer.

\section{Ocular irritancy test}

The prepared formulation i.e. in-situ gel was evaluated for ocular irritancy in Albino Rabbits $(n=3)$. They were housed and maintained according to protocol. Eyes were marked as test and control respectively. The control eyes received no sample while the test eyes received the formulation $(0.5 \mathrm{ml})$ and observed for the ocular irritancy including the macroscopic observation of cornea, iris and conjunctiva. The protocol was approved by IAEC of the institute in accordance with CPCSEA norms (Lic No.- (1205/C/08/CPCSEA/21.04.08).

\section{Ex-vivo drug release study of Nano particulate in- situ gel}

Goat cornea was used for the present investigation to study permeation across the corneal membrane. Whole eyeballs of goat were procured from a slaughterhouse and transported to laboratory in cold condition in normal saline maintained at $4^{\circ} \mathrm{C}$.

The cornea were carefully removed along with a 5-6 $\mathrm{mm}$ of surrounding scleral tissue and washed with cold saline. The washed cornea were kept in cold and freshly prepared solution of STF ( $\mathrm{pH}$ 7.4). The study was carried out by using Franz-diffusion cell in such a way that corneal side continuously remained in an intimate contact with the formulation in the donor compartment. The receptor compartment was filled with STF $(\mathrm{pH}$ 7.4 at $\left.34^{\circ} \mathrm{C} \pm 0.5^{\circ} \mathrm{C}\right)$. The receptor medium was stirred on a magnetic stirrer. The samples were withdrawn at different time intervals and analyzed for drug content. Receptor phase were replenished with an equal volume of STF (pH 7.4) at each time interval. ${ }^{13}$

\section{Accelerated stability studies}

Formulations were packed in amber colour vials and sealed with aluminum foil and stored at $40 \pm 2^{\circ} \mathrm{C}$ and 75 $\pm 5 \% \mathrm{RH}$ for 3 months as per ICH guidelines. Sample was analyzed at specific time intervals for clarity, $\mathrm{pH}$ and in-vitro dissolution. ${ }^{14}$ 


\begin{tabular}{|c|c|c|c|c|c|c|}
\hline S. No. & Drug & \multirow[t]{2}{*}{ Batch code } & \multicolumn{2}{|c|}{ Polymer (mg) } & \multirow[t]{2}{*}{ Gelling capacity } & \multirow[t]{2}{*}{ Viscosity (cP) } \\
\hline \multirow[t]{8}{*}{1.} & Ofloxacin & & HPMC K100M & Carbopol 940 & & \\
\hline & & F1 & 50 & 450 & + & 413 \\
\hline & & F2 & 100 & 650 & ++ & 668 \\
\hline & & F3 & 150 & 850 & +++ & 860 \\
\hline & & & \multicolumn{2}{|c|}{ Gelrite } & & \\
\hline & & F4 & \multicolumn{2}{|c|}{500} & ++ & 643 \\
\hline & & F5 & \multicolumn{2}{|c|}{750} & +++ & 862 \\
\hline & & F6 & \multicolumn{2}{|c|}{1000} & +++ & 1080 \\
\hline
\end{tabular}

Notice: (-) no gelation, (+) gel after a few minutes dissolved rapidly, (++) immediate gelation that remained for few hours and (+++) immediate gelation that remained for extended period.

Table 2: Formulation design of nanoparticulate insitu gel $(100 \mathrm{ml}, 0.3 \%)$.

\begin{tabular}{|c|c|}
\hline Ingredients (mg) & Amount (w/v) \\
\hline Ofloxacin nanoparticles (mg) & 1020.1 \\
\hline Gellan gum (mg) & 750 \\
\hline Benzalkonium chloride (\%) & 0.1 \\
\hline $\mathrm{NaOH}(0.1 \mathrm{M})$ & q.s for pH adjustment \\
\hline Purified water & q.s upto $100 \mathrm{ml}$ \\
\hline
\end{tabular}

\section{RESULTS AND DISCUSSION}

In the present study, Nano particulate in-situ gel of Ofloxacin was prepared to increase the residence time of drug on ocular surface which is a major drawback of majority of conventional dosage forms i.e. eye drops, ointments and gels etc. Fluoroquinolones are commonly used antimicrobials (effective for both gram negative and gram positive bacteria) in the treatment of various bacterial infections and are generally well tolerated. ${ }^{15}$ Ofloxacin, a fluoroquinolone drug is used for the treatment of conjunctivitis and corneal ulcers and it is given quarterly in a day because its bioavailability is very low. Hence, nanoparticles of Ofloxacin were firstly prepared using EC to improve the bioavailability of drug. The stock solution of Ofloxacin $(5 \mu \mathrm{g} / \mathrm{ml})$ was prepared using $\mathrm{pH} 7.4$ phosphate buffer and scanned between $200-400 \mathrm{~nm}$ which concluded $\lambda_{\max }$ of $295 \mathrm{~nm}$ (Figure 1). Compatibility of drug with different excipients was checked by FTIR and TLC densitometric studies. The IR spectra, of pure drug alone and in its combination with the polymers used, were obtained and compared and depicted in following Figures (Figure 2). FTIR spectra of the pure OFL and the drug polymer mixture showed characteristic bands at $3000 \mathrm{~cm}^{-1}$ (stretching vibration of $\mathrm{OH}$ group, $\mathrm{NH}$ stretching), 2520.51 $\mathrm{cm}-1$ and $2135.78 \mathrm{~cm}^{-1}(\mathrm{vCH} 3 \mathrm{gp}), 1803.12 \mathrm{~cm}^{-1}$ (acidic carbonyl $\mathrm{C}=\mathrm{O}$ stretching) and 1464.67 (stretching vibration of $\mathrm{CH} 2$ ), indicating the chemical stability $\mathrm{OFL}$ in the chosen polymeric mixture. Similar results were presented by Kumar $\mathrm{M}$, et al. ${ }^{16}$ The $\mathrm{R}_{f}$ values of pure Ofloxacin and with various excipients ranged between 0.62-0.729 (Table 3 and Figure 3).

Prepared nanoparticles were evaluated for Percentage yield, Drug entrapment efficiency (EE), Particle size analysis, Zeta potential analysis and ex-vivo permeation (Table 4). Results of in-vitro dissolution profile in Simulated Tear Fluid (STF) have depicted (Figure 5). The in-vitro release studies conducted on nanoparticles indicated that the OFL loaded ethyl cellulose nanoparticles provided sustained drug release over a period of $12 \mathrm{hr}$. Whole experimental data was fitted into $3^{2}$ full factorial design and results of regression analysis interpreted that both independent variables i.e. the amount of EC (factor A) and percentage of PVA (factor B) had positive effect on dependent variables i.e. particle size and entrapment efficiency with $p$ value of 0.0001 and 0.0090 respectively. Results of factorial design were as shown in following table (Table 5, 6). It was observed from equation (1) (Table 6) that A and B factors had significant positive effect on Particle size, which indicated that when the values of $\mathrm{A}$ and $\mathrm{B}$ increased, the response increased $(p=0.0001)$ i.e. as the amount of EC and percentage of PVA was increased, Particle size and Entrapment efficiency were also increased. Elaissari A, et al. ${ }^{17}$ reported that there was no considerable influence of different concentrations of PVA on the mean particle size. However, after a certain limit of concentration of PVA (above $0.1 \mathrm{mg} / \mathrm{mL}$ ) a large increase in particle size was observed. This sudden increase in size could be attributed to deposition of extra PVA onto the surface of particles.

It was observed from equation (1) (Table 6) that both A and B factors also had significant positive effect on Entrapment efficiency, which indicated that when the values of $\mathrm{A}$ and $\mathrm{B}$ increased, the response increased 


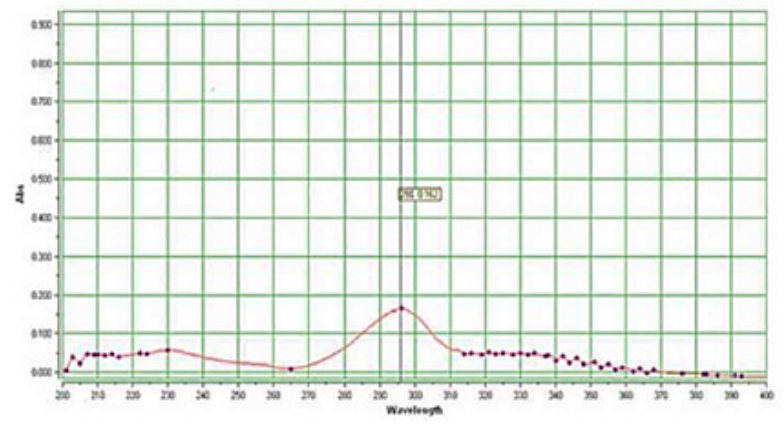

Figure 1: UV scan of Ofloxacin showing characteristic wavelength.
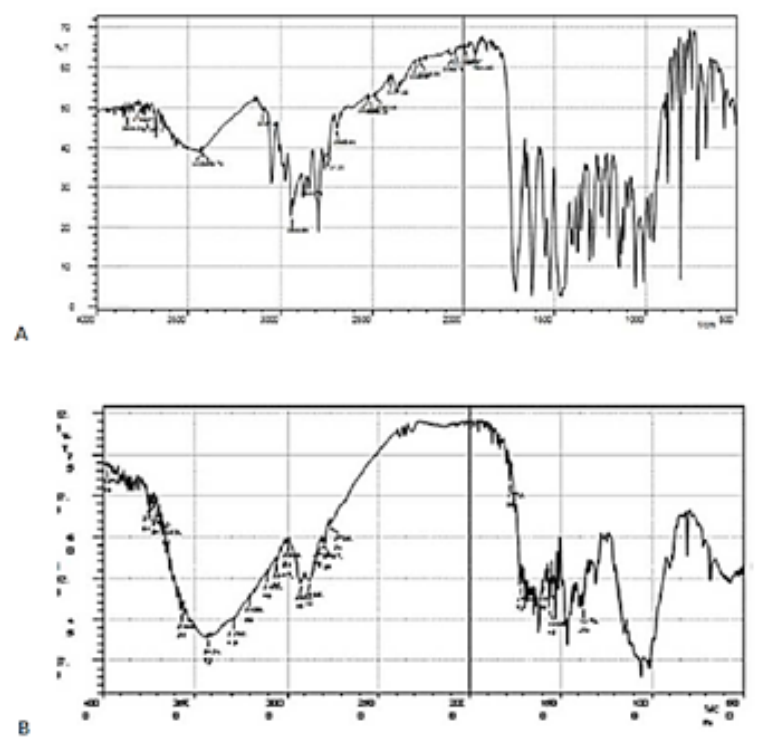

Figure 2: FTIR spectra of A) Ofloxacin (pure drug), B) Ofloxacin with Gellan gum.

$(p=0.0090)$ and hence, both dependent variables were increased with increase in independent variables.

From Response surface plots (Figure 6,7), it was concluded that both particle size and entrapment efficiency increased from blue to orange region owing to increase in amount of EC and PVA.

Optimization study thus conducted, has given a predicted solution. Predicted solution had the similar composition (factor A: $300 \mathrm{mg}$; factor B: $2 \%$ ) and showed identical responses as that of batch B3 and thus it was selected as optimized batch with a desirability of 1 . The optimized batch (B3) showed particle size (148 nm, Figure 4$), \mathrm{EE}(57.22 \%)$ and drug release $(52.5 \%$ and $51.3 \%$ ) in $\mathrm{pH} 7.4$ phosphate buffer and simulated tear fluid, respectively. Release kinetic study showed that drug release had followed korsemeyer peppas model. The values of $R^{2}$ and $n$ were found to be 0.949 and 0.646 respectively that showed anomalous transport as a mechanism of drug release.

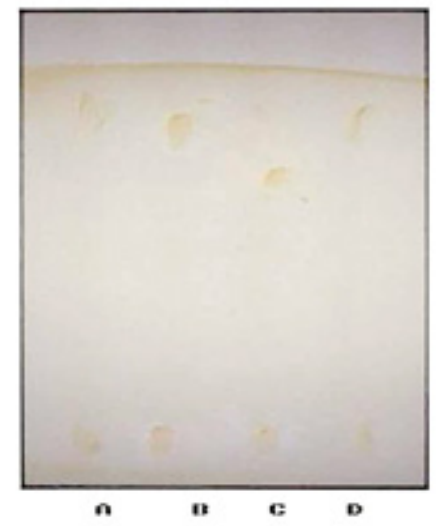

Figure 3: Photographic representation of TLC with different drug polymer combination.

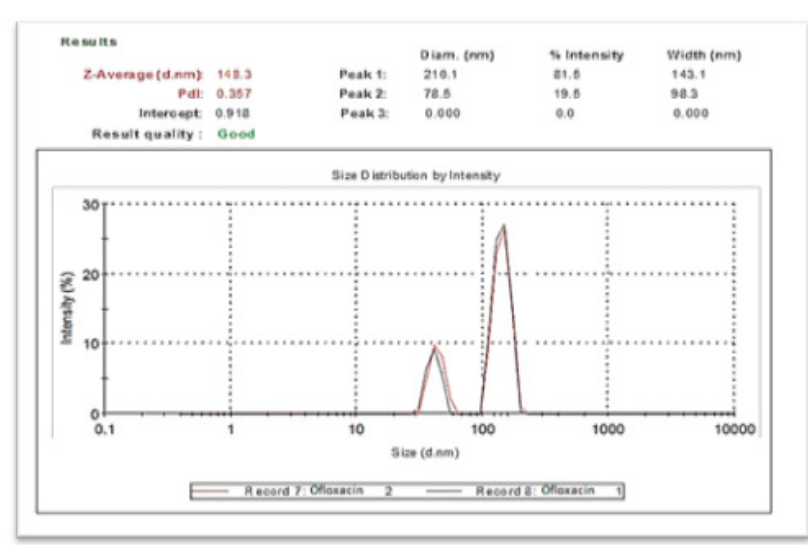

Figure 4: Particle size analysis of Ofloxacin nanoparticles (Optimized batch B3).

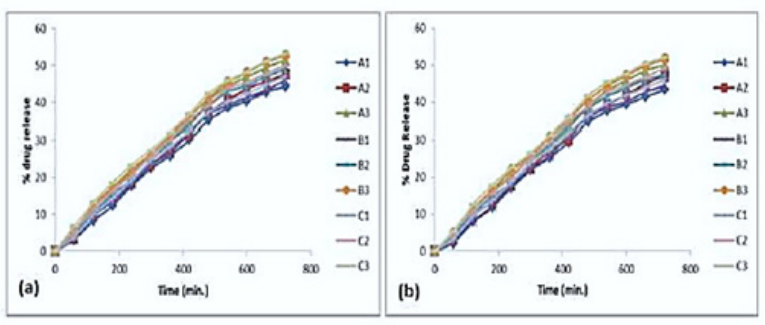

Figure 5: In-vitro dissolution of nanoparticles in (a) pH 7.4 phosphate buffer and (b) simulated tear fluid.

\begin{tabular}{|c|c|c|}
\hline \multicolumn{3}{|c|}{ Table 3: $\mathbf{R}_{\boldsymbol{f}}$ value of different combination of drug and } \\
polymer. \\
\hline S. No. & Ingredients & $\mathbf{R}_{\boldsymbol{f}}$-value \\
\hline A & Ofloxacin & 0.720 \\
\hline B & Ofloxacin: Ethyl Cellulose & 0.729 \\
\hline C & Ofloxacin: Carbopol & 0.623 \\
\hline D & Ofloxacin: Gallen gum & 0.722 \\
\hline
\end{tabular}




\section{Table 4: Various evaluative parameters of Ofloxacin nanoparticles.}

\begin{tabular}{|c|c|c|c|c|}
\hline \multirow{2}{*}{ Formulation code } & \multicolumn{4}{|c|}{ Evaluation Parameters } \\
\cline { 2 - 5 } & Percentage yield & $\begin{array}{c}\text { Drug entrapment efficiency } \\
(\%)\end{array}$ & Particle size analysis (nm) & Zeta potential analysis \\
\hline A1 & 35.5 & 40.52 & 152 & -20.65 \\
\hline A2 & 45.73 & 49.69 & 156 & -19.72 \\
\hline A3 & 58.67 & 56.92 & 160 & -14.35 \\
\hline B1 & 36.25 & 43.75 & 139 & -17.59 \\
\hline B2 & 48.1 & 51.55 & 144 & -8.78 \\
\hline B3 & 59.10 & 57.22 & 148 & -22.8 \\
\hline C1 & 37.06 & 48.37 & 168 & -11.55 \\
\hline C2 & 46.83 & 55.14 & 174 & -7.56 \\
\hline C3 & 58.96 & 58.74 & 180 & -2.83 \\
\hline
\end{tabular}

\begin{tabular}{|c|c|c|c|c|}
\hline \multirow[t]{2}{*}{ Formulation Code } & \multicolumn{2}{|c|}{ Variable levels in coded form } & \multirow{2}{*}{$\begin{array}{l}\text { Particle size } \\
\qquad(\mathrm{nm})\end{array}$} & \multirow{2}{*}{$\begin{array}{c}\text { Entrapment } \\
(\%)\end{array}$} \\
\hline & X1 (mg) & $\mathrm{X} 2(\%)$ & & \\
\hline A1 & -1 & -1 & 152 & 40.52 \\
\hline A2 & 0 & -1 & 156 & 49.69 \\
\hline A3 & 1 & -1 & 160 & 56.92 \\
\hline B1 & -1 & 0 & 139 & 43.75 \\
\hline B2 & 0 & 0 & 144 & 51.55 \\
\hline B3 & 1 & 0 & 148 & 57.22 \\
\hline C1 & -1 & 1 & 168 & 48.37 \\
\hline $\mathrm{C} 2$ & 0 & 1 & 174 & 55.14 \\
\hline C3 & 1 & 1 & 180 & 58.74 \\
\hline \multirow[t]{2}{*}{ Coded value } & \multicolumn{2}{|c|}{ Actual value } & & \\
\hline & $\mathrm{X} 1(\mathrm{mg})$ & $\mathrm{X} 2(\%)$ & & \\
\hline-1 & 100 & 1 & & \\
\hline 0 & 200 & 2 & & \\
\hline 1 & 300 & 3 & & \\
\hline
\end{tabular}

\section{Table 6: Summary of regression analysis results.}

\begin{tabular}{|c|c|c|c|c|c|c|}
\hline \multicolumn{7}{|c|}{ For particle size } \\
\hline \multicolumn{2}{|c|}{ Polynomial equation } & \multicolumn{4}{|c|}{$Y=143.78+4.83 A+9.00 B+1.00 A B-0.17 A^{2}+21.33 B^{2}$} & eq (1) \\
\hline Response & Bo & A & B & $A B$ & $A^{2}$ & $\mathrm{~B}^{2}$ \\
\hline FM & +143.78 & +4.83 & +9.00 & +1.00 & -0.17 & +21.33 \\
\hline$p$-value & \multicolumn{6}{|c|}{0.0001} \\
\hline \multicolumn{7}{|c|}{ For entrapment efficiency } \\
\hline \multicolumn{2}{|c|}{ Polynomial equation } & \multicolumn{4}{|c|}{$Y=51.67+6.73 A+2.52 B-1.51 A B-1.19 A^{2}+0.69 B^{2}$} & eq (2) \\
\hline Response & Bo & A & B & $A B$ & $A^{2}$ & $\mathrm{~B}^{2}$ \\
\hline FM & +51.67 & +6.73 & +2.52 & -1.51 & -1.19 & +0.69 \\
\hline$p$-value & \multicolumn{6}{|c|}{0.0090} \\
\hline
\end{tabular}




\begin{tabular}{|c|c|c|c|c|c|}
\hline \multirow{2}{*}{ S. No. } & \multirow{2}{*}{ Drug } & \multicolumn{4}{|c|}{ Table 7: Evaluation Parameters of Optimized batch (B3). } \\
\cline { 3 - 6 } & & Percentage yield & \% Entrapment Efficiency & Particle size (nm) & Zeta Potential \\
\hline 1. & Ofloxacin & 59.10 & 57.22 & 148.0 & -22.8 \\
\hline
\end{tabular}

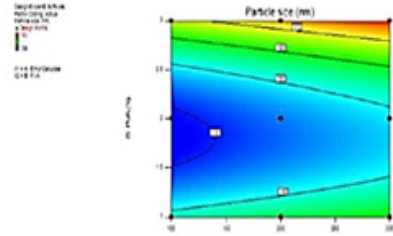

A

Figure 6: 3D Response Surface plot showing the influence of ethyl cellulose and PVA on Particle size.

\begin{tabular}{|c|c|c|}
\multicolumn{3}{|c|}{ Table 8: Evaluation parameter of nanoparticulate in- } \\
situ ocular gel of Ofloxacin.
\end{tabular}

\begin{tabular}{|c|c|c|}
\hline Time (min.) & $\begin{array}{c}\text { pH 7.4 Phosphate } \\
\text { buffer Saline (PBS) }\end{array}$ & $\begin{array}{c}\text { Simulated Tear Fluid } \\
\text { (STF) }\end{array}$ \\
\hline 0 & 0 & 0 \\
\hline 60 & 9.57 & 8.62 \\
\hline 120 & 16.55 & 15.74 \\
\hline 180 & 20.66 & 19.32 \\
\hline 240 & 24.35 & 23.85 \\
\hline 300 & 28.38 & 27.36 \\
\hline 360 & 34.65 & 33.62 \\
\hline 420 & 39.49 & 38.49 \\
\hline 480 & 44.32 & 43.33 \\
\hline 540 & 48.83 & 47.82 \\
\hline 600 & 52.79 & 51.77 \\
\hline 660 & 55.71 & 54.61 \\
\hline 720 & 62.19 & 61.82 \\
\hline
\end{tabular}

Various evaluative parameters obtained for optimized batch (B3) were as follows (Table 7 and Figure 8-10). Zeta potential analysis of ofloxacin nanoparticles belonging to optimized batch i.e B3 peformed on Malvern zeta sizer were as follows (Figure 8). The surface
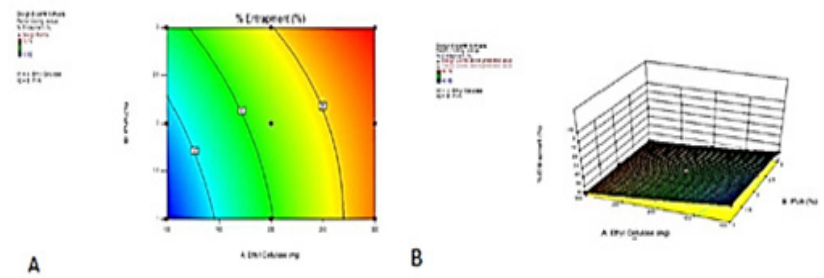

Figure 7: 3D Response Surface plots showing the influence of ethyl cellulose and PVA on Entrapment efficiency.
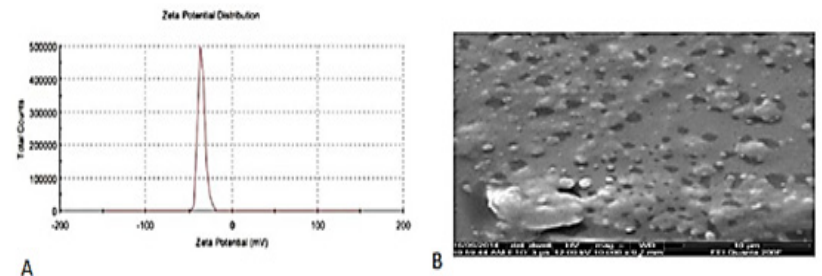

Figure 8: A) Zeta potential of optimized batch (B3), B) SEM image of nanoparticles of Optimized formulation (B3) (5000X magnification).

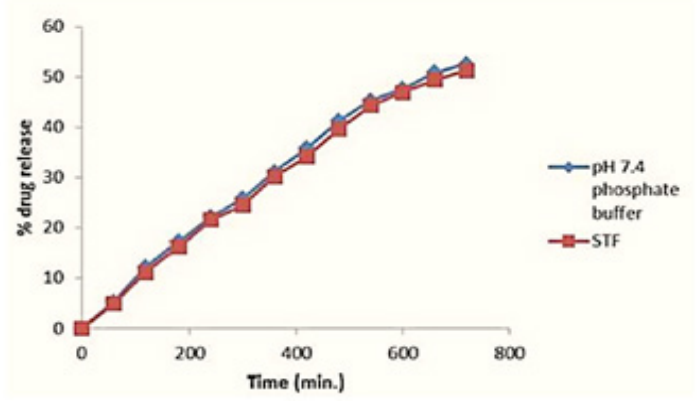

Figure 9: In-vitro release study of optimized batch of Ofloxacin nanoparticles (B3) in pH 7.4 Phosphate buffer and Simulated Tear Fluid (STF).

morphology of ofloxacin nanoparticles belonging to the significant batch B3 was examined by SEM (Figure 8). In-vitro dissolution study was conducted on optimized batch i.e. B3 in pH 7.4 Phosphate buffer and Simulated Tear Fluid (Figure 9). Results of kinetic studies were presented in Figure 10.

Optimized batch was then used to formulate in-situ gel using gellan gum, selected by comparing gelling capacities of different gelling agents. The mechanism of gelation by gellan gum involves the formation of double helical junction zones followed by aggregation of 

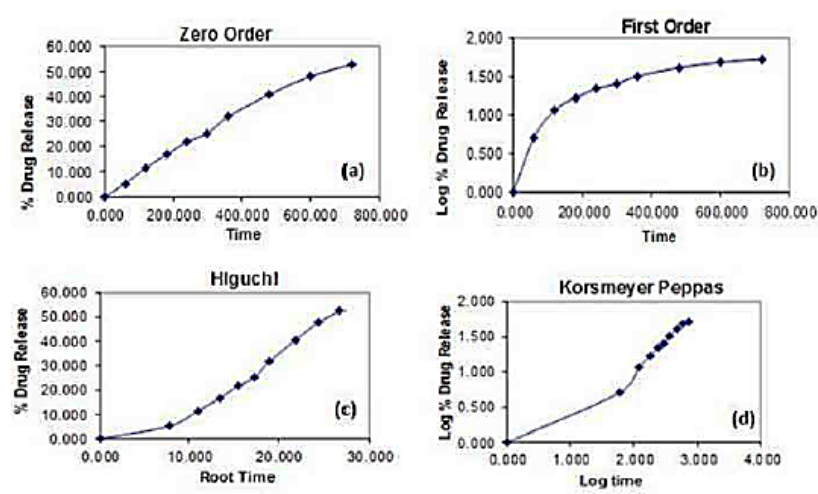

Figure 10: Release kinetics graphs- a. Zero order, b. First order, c. Higuchi model and d. Korsemeyer peppas models of optimized batch (B3) in Simulated Tear Fluid (STF).

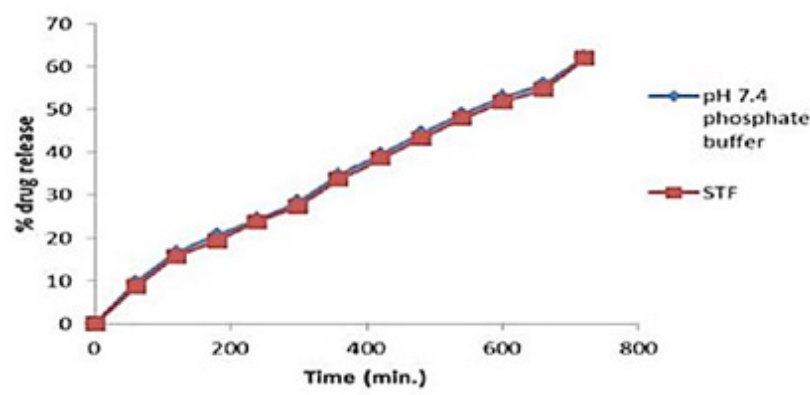

Figure 11: Comparative ex-vivo release of Ofloxacin nanoparticulate in-situ ocular gel in pH 7.4 phosphate buffer and Simulated Tear Fluid (STF).

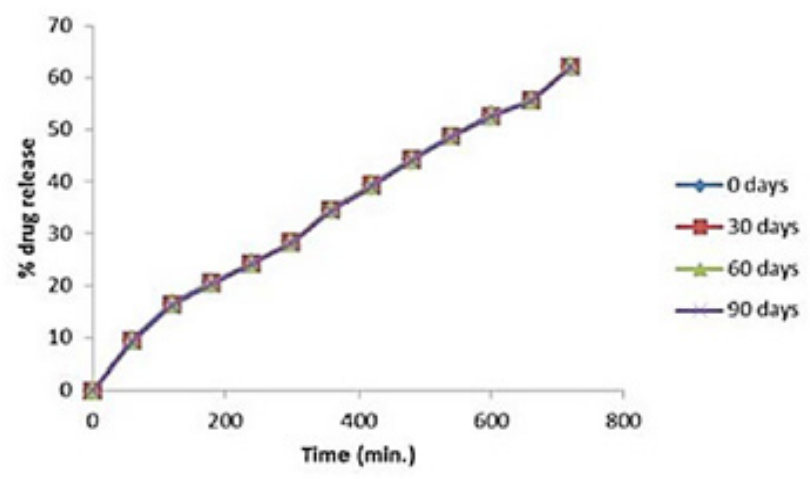

Figure 12: Comparative release profile of Ofloxacin nanoparticulate in-situ ocular gel on stability studies

double helical segment to form three dimensional networks by complexation with cations and hydrogen bonding with water which consequently prolongs the residence time of drug at absorption site and increases the bioavailability of drug. It is widely used in ophthalmology because of its thixotropic, thermo plasticity and pseudo plasticity.

Nano particulate in-situ gel of Ofloxacin thus prepared was evaluated for various parameters i.e. clarity, $\mathrm{pH}$, viscosity, gelling strength, in-vitro diffusion and stability studies (Table 8, 9 and Figure 11,12). The in-vitro drug release from Nano particulate in-situ ocular gel was found to be $62.19 \%$ and $61.82 \%$ in $\mathrm{pH} 7.4$ phosphate buffer and simulated tear fluid (STF), respectively, over a period of $12 \mathrm{~h}$. Results of ex-vivo diffusion study were depicted in Table 9 and Figure 11. During stability studies, samples were collected at specified time intervals and evaluated for release characteristics (Figure 12).

\section{CONCLUSION}

In the present study, Nano particulate in-situ gel of OFL was prepared for the treatment of conjunctivitis and corneal ulcers by utilizing the previously prepared nanoparticles. Initially, nanoparticles of Ofloxacin were prepared and evaluated for various evaluation parameters. Full factorial design was adopted to optimize the formulation and based on the predicted solution given by the software a checkpoint batch (B3) was selected and considered as an optimized batch. Nano particulate insitu gel was prepared with the optimized batch and prepared gel was further evaluated for various parameters and satisfactory results were obtained. Nano particulate in-situ gel passed the irritancy test and found compatible. It was observed that an adequate amount of gel was permeated through the ocular tissue as confirmed by the ex-vivo study. Hence, it was concluded that the research work, in reference, comprised of quite novel approaches of investigations.

\section{ACKNOWLEDGEMENT}

The corresponding author acknowledges the Department of Pharmacy, Teerthanker Mahaveer University for conducting the research work.

\section{CONFLICT OF INTEREST}

The authors report no conflicts of interest.

\section{ABBREVIATIONS}

OFL: Ofloxacin; STF: Simulated Tear Fluid; TLC: Thin Layer Chromatography; FTIR: Fourier Transmission Infra-Red; EC: Ethyl Cellulose; PVA: Poly Vinyl Alcohol; SEM: Scanning Electron Microscopy; ICH: International Conference of Harmonization; PBS: Phosphate buffer Saline; RH: Relative humidity, $\mathbf{R}_{\boldsymbol{f}}$ Retention factor.

\section{REFERENCES}

1. Bhowmik D, Gopinath H, Pragati BK, Duraivel S, Sampath KP. Controlled Release Drug Delivery System. The Pharma Innovation. 2012;1(10):24-32. 
2. Sharma UK, Verma A, Prajapati SK, Pandey H. Ocular drug delivery: Assorted obstructions and contemporary progress. Int J Res Dev Pharm Life Sci. 2013;2(2):464-73.

3. Salata OV. Application of nanoparticles in biology and medicine. J Nanobiotechnol. 2004;2(1):1-6.

4. Garg A, Visht SS, Sharma PK, Kumar N. Formulation, Characterization and Applications of Nanoparticles. Der Pharmacia Sinica. 2011;2(2):17-26.

5. Nerkar TS, Gujarathi NA, Bhushan R, Bakliwal SR, Pawar SP. In-situ gel: Novel approach in sustained and controlled drug delivery system. Pharma Sci Monit: An Int J Pharm Sci. 2013;4:1-18.

6. https://www.drugs.com/cdi/ofloxacin-drops.html. [Last updated on 2016 July 7; Last cited on 2016 July 11].

7. Attimarad MV, Ahmed OA. A conventional HPLC-MS method for the simultaneous determination of Ofloxacin and Cefixime in plasma: Development and validation. J of Basic and Clin Pharmacy. 2013;4(2):36-41.

8. https://www.medicinenet.com/ofloxacin ophthalmic solution/article.htm. [Last updated on 2016 May 10; Last cited on 2016 July 15]

9. Gandhimathi M, Ravi TK, Nilima S. Validated high performance thin layer chromatography method for simultaneous estimation of Ofloxacin and Ornidazole in tablet dosage form. IJPS. 2006;68(6):838.
10. Yuan H, Xuefan X, Qingyu X, Zhiyao H, Yuehua L, Dan Z. Crystalline drug aconitine-loaded poly (d,I-Lactide-co glycolide) nanoparticles preparation and in-vitro release. J Pharm Soc Jpn. 2010;130(3):409-18.

11. Devi KV, Bhosale UV. Formulation and Optimization of polymer nano drug delivery system of Acyclovir using 32 full factorial design. Int J Pharm Tech Res. 2009;1(3):644-53.

12. Vodithala S, Khatry S, Shastri N, Sadanandam M. Formulation and Evaluation of ion-activated ocular gels of ketorolac tromethamine. Int J Curr Pharm Res. 2010;2(3):33-8.

13. ICH Q1A (R) guidelines: Stability testing of New Drug Substance and Product. 2003;1-18.

14. Rathore KS. Development and in-vivo in-vitro characterizations of Timolol maleate in-situ gels. Intl J Pharma Biosci. 2011;2(3):248-63.

15. Kameswari PD, Selvaraj N, Mangaiarkkarasi A. Fixed drug eruptions caused by cross-reactive quinolones. J of Basic and Clin Pharmacy. 2014;5(2):54-5.

16. Manish K, Amit S, Pravin K, Anil K, Mayank C, Priyanka B. Formulation and in-vitro Evaluation of Periodontal Films Containing Ofloxacin. J Chr D D. 2011;2(1):37-41.

17. Waisudin B, Karim M, Rkia E, Hatem F, Abdelhamid E. Elaboration of Nanoparticles Containing Indomethacin: Argan Oil for Transdermal Local and Cosmetic Application. Journal of Nanomaterials. 2015;18:1-9.

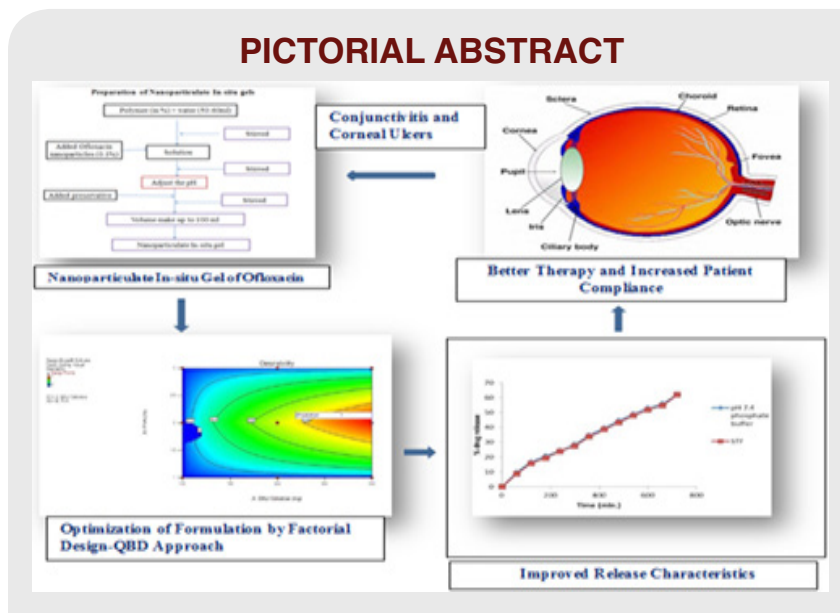

\section{SUMMARY}

- Ofloxacin (OFL) stops or prevents ocular bacterial infections by either killing or inhibiting their growth

- A serum concentration of Ofloxacin after 10 days of topical dose is very much less than the standard oral doses of the same

- Prepared in-situ gel would prolong the residence time of drug at corneal surface which is a crucial need for legitimate entry of drug in interior segment of eye for complete mending action.

\section{About Authors}

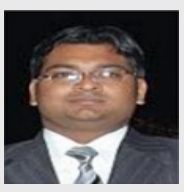

Dr. Akhil Sharma, M. Pharm., Ph.D., is presently working as Professor and Principal at R.J College of Pharmacy, Raipur, Aligarh, U.P., India. He has completed his Ph.D. in 2016 from Teerthanker Mahaveer University, Moradabad, U.P., India. He has been serving the profession of Pharmacy from more than 10 years. He has published articles in many peer reviewed journals.

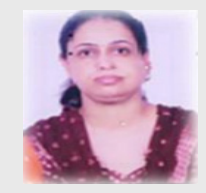

Dr. Shaweta Sharma, M. Pharm., Ph.D., is currently employed as Associate Professor of Pharmaceutics at Galgotias University, Yamuna Expressway, Gautam Bhuddha Nagar. She has completed Ph.D. in 2017 from Teerthanker Mahaveer University, Moradabad, U.P., India. She has been contributing to academics from more than 10 years. She has published articles in many peer reviewed journals.

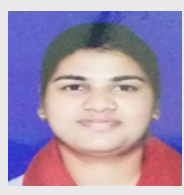

Ms. Dimple Singh Tomar, M.Pharm., Ph.D. (pursuing), is currently working as Senior lecturer at Kherval Subharti College of Pharmacy, Subharti University, Meerut, U.P. She is keenly interested in research activities.

Cite this article: Sharma A, Sharma S, Tomar DS. Optimization of in-situ Nanoparticulate Gel of Ofloxacin using Factorial Design to Improve Treatment Strategy for Conjunctivitis and Corneal Ulcers. Indian $\mathrm{J}$ of Pharmaceutical Education and Research. 2020;54(2):284-92. 